\title{
Exercise Completed When Young Provides Lifelong Benefit to Cortical Bone Structure and Estimated Strength
}

\author{
Stuart J. Warden, ${ }^{1,2}$ Sara Mantila Roosa, ${ }^{1}$ Andrea L. Hurd, ${ }^{1}$ Robyn K. Fuchs, ${ }^{1,2}$ \\ ${ }^{1}$ Center for Translational Musculoskeletal Research, Indiana University School of Health and \\ Rehabilitation Sciences; ${ }^{2}$ Department of Physical Therapy, Indiana University School of Health and \\ Rehabilitation Sciences
}

\begin{abstract}
Exercise induces greatest bone gains during growth, yet reduced bone strength is an age-related phenomenon. This raises the question of whether exercise-induced bone changes when young persist into adulthood. The current studies used Major/Minor League Baseball (MLB/MiLB) players to explore whether exercise-induced gains in humeral bone structure and strength accrued when young persist lifelong. MLB/MiLB players are a unique model as the unilateral upper extremity loading associated with throwing enables the contralateral side to serve as an internal control site and former MLB/MiLB players were consistently exposed to extreme loading reducing secular variations in exercise levels between generations. Dominant-to-nondominant (D-to-ND) differences in humeral cross-sectional properties in MLB/MiLB players were normalized to matched controls to correct for side-to-side differences due to elevated habitual loading associated with arm dominance.
\end{abstract}

Exercise when young induced significant skeletal benefits, with active MLB/MiLB players having nearly double the estimated ability to resist torsion (polar moment of inertia, $\mathrm{I}_{\mathrm{P}}$ ) in the humerus of their dominant arm. The cortical bone mass and area benefits of exercise observed in active MLB/MiLB players were lost in former MLB players following 40-49 years of detraining as a result of elevated medullary expansion and endocortical trabecularization. However, $42 \%$ of the total bone area benefit persisted following $50+$ years of detraining and contributed to the maintenance of $24 \%$ of the benefit on $I_{P}$. In MLB players who continued to exercise during aging, medullary expansion and endocortical trabecularization were reduced and there was maintenance of the cortical bone mass and area benefits of exercise.

These cumulative data indicate: 1) the extreme plasticity of the growing skeleton to exercise; 2) that exercise when young has lifelong benefits on cortical bone size and estimated strength, but not bone mass, and; 3) exercise continued during aging maintains the bone mass benefits of exercise. 